\title{
The Impact of Adjuvant Management Strategies on Outcomes in Women With Early Stage Uterine Serous Carcinoma
}

\author{
Andrew Cook ${ }^{1}$, Remonda Khalil ${ }^{2}$, Charlotte Burmeister ${ }^{2}$, Irina Dimitrova ${ }^{3}$, Mohamed A. Elshaikh ${ }^{1}$ \\ 1. Radiation Oncology, Henry Ford Health System, Detroit, USA 2. Biostatistics, Henry Ford Health System, Detroit, \\ USA 3. Gynecologic Oncology, Henry Ford Health System, Detroit, USA
}

Corresponding author: Mohamed A. Elshaikh, melshai1@hfhs.org

\section{Abstract \\ Objective}

To determine the impact of different adjuvant strategies on outcomes in women with early-stage uterine serous carcinoma (USC).

\section{Methods}

Our retrospective database for women with endometrial carcinoma was queried for women with 2009 International Federation of Gynecology and Obstetrics (FIGO) stages I-II USC who underwent surgical staging between January 1991 and April 2019 followed by adjuvant management (observation, radiation therapy (RT), chemotherapy (CT), or combined modality treatment (CRT)). Chi-square tests were performed to compare differences in outcome by type of adjuvant management. Recurrence-free survival (RFS), disease-specific survival (DSS), and overall survival (OS) were assessed by Kaplan-Meier and log-rank tests. Univariate and multivariate analyses (MVA) were performed to identify statistically significant predictors of survival endpoints.

\section{Results}

We identified 171 women who met our inclusion criteria. The median follow-up time was 70.5 months. Seventy-five percent of the study cohort was FIGO stage IA, $13 \%$ were stage IB, and $12 \%$ were stage II. All women underwent pelvic lymph node dissection with a median number of dissected lymph nodes of 14 . Omentectomy was performed in $64 \%$ of patients. Adjuvant RT was utilized in $56 \%$ of women (65 patients received vaginal brachytherapy alone, 10 patients received pelvic RT, and 21 patients received a combination of both). The most commonly used chemotherapy regimen was carboplatin and paclitaxel with a median number of cycles of six. A total of $44 \%$ of the cohort received CRT, $12 \%$ received RT alone, $19 \%$ received chemo alone, and $25 \%$ were observed. Five-year RFS was $73 \%$ for those who received CRT, $84 \%$ for those who received RT alone, $68 \%$ for those who received CT alone, and $55 \%$ for those who were observed $(p=0.13)$. Five-year DSS was $81 \%, 94 \%, 71 \%$, and $60 \%$, respectively $(p=0.02)$. Five-year OS was $76 \%, 70 \%, 60 \%$, and $56 \%$, respectively $(p=0.11)$. On MVA of OS and DSS, a higher percentage of myometrial invasion, the

Review began 02/10/2021 Review ended 02/16/2021 Published 02/23/2021

\section{() Copyright 2021}

Cook et al. This is an open access article distributed under the terms of the Creative Commons Attribution License CC-BY 4.0., which permits unrestricted use, distribution, and reproduction in any medium, provided the original author and source are credited. presence of lower uterine segment involvement, positive peritoneal cytology, and receipt of chemotherapy alone/observation were independent predictors of worse outcomes. The sole independent predictor of worse RFS on MVA was the presence of positive peritoneal cytology.

\section{Conclusion}

In this cohort of women with early-stage USC who underwent surgical staging, adjuvant radiation treatment with or without chemotherapy was associated with improved survival endpoints and trended toward improved recurrence rates.

Categories: Obstetrics/Gynecology, Radiation Oncology, Oncology

Keywords: endometrial carcinoma, serous carcinoma, adjuvant radiation therapy, hysterectomy, hysterectomy , adjuvant chemotherapy

\section{Introduction}

Endometrial cancer remains the most commonly diagnosed gynecologic malignancy in the United States [1]. Women with early-stage endometrial cancer have a favorable prognosis, with five-year survival rates of around $85 \%$ [2]; however, there remains a subset of $10 \%$ of patients who are diagnosed with uterine serous carcinoma (USC), which is known to have aggressive biology [3-4]. These patients have poorer outcomes in terms of recurrence and survival rates [5-10]. Although patients with USC comprise less than $15 \%$ of patients with endometrial carcinoma, they cause $40 \%$ of endometrial cancer deaths [11]. Furthermore, patients with early-stage USC constitute a heterogenous population with various risk factors. Studies for women with early-stage USC have found that deep myometrial invasion and positive peritoneal cytology are independent 
predictors of mortality [12-14]. Additional factors that may portend a poor prognosis for early USC are cervical stromal invasion and lymphovascular space invasion [15-16]. Outside of pathologic factors, African American patients with endometrial cancer have been found to have higher mortality rates [17].

Surgical staging is the cornerstone in the management of women with USC. Knowing that there is a high risk of recurrence in these patients, there is interest in optimizing adjuvant therapy. Given the low numbers of patients with early USC, it is difficult to accrue patients solely with USC histology to prospective randomized trials, and retrospective evidence has been necessary to guide treatment paradigms, albeit with conflicting reported outcomes.

While a recent study showed that adjuvant chemotherapy (chemo) with or without radiotherapy (RT) is associated with improved survival [18], several studies show that both chemo and RT are associated with improved survival outcomes compared to adjuvant chemo alone or RT alone in women with USC [19-21]. A large meta-analysis suggested a survival benefit to combined chemo and RT (CRT) for patients with early USC [22]. All of these adjuvant strategies, including observation, are valid approaches for early-stage USC according to the most recent National Comprehensive Cancer Network Uterine Neoplasms guidelines (version 1.2021).

These retrospective studies have been helpful in guiding adjuvant treatment; however, they are hampered with limitations that make them difficult to apply to clinical practice. These problems entail examining strictly non-myoinvasive USC [18], including advanced stages [22], and excluding patients who received pelvic RT [20]. Several studies also included patients with clear cell carcinoma [20-21,23]; clear cell carcinoma is thought to behave differently than USC and carries a different prognosis [24]. Additionally, some of these studies include analyses of large national databases that lack data on tumor recurrence [19].

Given that data remain conflicting on the optimal treatment for this subset of patients, the current study aimed to examine the outcomes of women with early-stage USC who underwent surgical staging followed by different adjuvant management approaches.

This research was earlier presented at the annual American Society for Radiation Oncology (ASTRO) meeting on October 24, 2020.

\section{Materials And Methods}

After institutional review board approval, our retrospective database was queried for patients with 2009 International Federation of Gynecology and Obstetrics (FIGO) stages I and II USC between the ages of 18 and 90 (age at hysterectomy). All patients were surgically staged between January 1991 and April 2017 and had at least one year of follow-up. Surgical staging was performed by a gynecologic oncologist with hysterectomy, salpingo-oophorectomy, with or without pelvic and para-aortic node dissection, and with or without omentectomy and peritoneal washings. These patients were divided into four groups based on adjuvant management received: observation, RT alone, chemo alone, or CRT. Patients with FIGO stage III-IV disease, non-serous endometrial histologies, synchronous malignancies, and non-operative candidates were excluded from this study.

Clinicopathologic factors that were evaluated in this study included patient age (as a continuous variable), body mass index (BMI), race, Charlson comorbidity score at the time of hysterectomy, percent myometrial invasion, FIGO stage, number of total lymph nodes (LN) examined (pelvic and paraaortic), peritoneal cytology status, lower uterine segment involvement, and lymphovascular space invasion (LVSI).

Survival curves were generated for the different adjuvant management groups using the Kaplan-Meier product-limit method calculated from the date of surgery. These were compared using the log-rank test to determine the rates of recurrence-free survival (RFS), disease-specific survival (DSS), and overall survival (OS). After univariate analysis, Cox regression analysis was performed to determine independent prognostic factors for RFS, DSS, and OS. Additionally, a backward selection process was used to restrict each of the models to contain all significant predictors. The Kruskal-Wallis and chi-square tests were used to determine significant differences $(\mathrm{p}<0.05)$ in various clinical factors amongst the groups. All analyses were performed using SAS version 9.4 (SAS Institute, Inc., Cary, NC).

\section{Results}

We identified 171 patients who met our inclusion and exclusion criteria. Median follow-up time was 70.5 months. None of the patients had residual disease post-hysterectomy. Thirty-five women (20\%) were diagnosed with non-myoinvasive FIGO stage IA USC. There were 104 patients (61\%) who underwent omentectomy as part of their surgical staging. Seventy-five patients (44\%) received adjuvant CRT, 42 patients (25\%) were followed with observation, 33 patients (19\%) received adjuvant chemo alone, and 21 patients (12\%) received adjuvant RT alone. For patients in the observation cohort, the reason why adjuvant therapy was not given was not clearly documented in the medical record; however, it is likely that most of these patients declined additional treatments. Table 1 summarizes information on patient characteristics amongst the groups, which were overall well-balanced. No statistically significant differences were noted 
between the groups except that a higher proportion of patients with FIGO stage IA disease were followed by observation after hysterectomy, and a higher proportion of patients in the CRT group underwent complete surgical staging with paraaortic lymph node dissection, omentectomy, and a higher median number of lymph nodes were examined.

\begin{tabular}{|c|c|c|c|c|c|}
\hline Variable & $\begin{array}{l}\text { No Adjuvant Treatment } \\
(\mathrm{N}=42)\end{array}$ & $\begin{array}{l}\text { RT Alone } \\
(\mathrm{N}=21)\end{array}$ & $\begin{array}{l}\text { Chemo Alone } \\
(\mathrm{N}=33)\end{array}$ & CRT (N=75) & $\mathbf{p}$ \\
\hline $\begin{array}{l}\text { Age at hysterectomy, years, median } \\
\text { (range) }\end{array}$ & $67.5(54-90)$ & $71(57-90)$ & $68(51-85)$ & $66(51-87)$ & 0.26 \\
\hline Median BMI, kg/m², median (range) & $30.3(19.6-54.3)$ & $\begin{array}{l}36.3(17.9- \\
48.7)\end{array}$ & $33.3(22.5-64.1)$ & $\begin{array}{l}32.3(18.3- \\
52.8)\end{array}$ & 0.08 \\
\hline \multicolumn{6}{|l|}{ Race, n (\%) } \\
\hline White & $21(50 \%)$ & $11(52 \%)$ & 17 (52\%) & $38(51 \%)$ & 0.97 \\
\hline African American & $19(45 \%)$ & $10(48 \%)$ & $16(48 \%)$ & $35(47 \%)$ & \\
\hline Other & $2(5 \%)$ & $0(0 \%)$ & $0(0 \%)$ & $2(3 \%)$ & \\
\hline \multicolumn{6}{|l|}{2009 FIGO staging, n (\%) } \\
\hline IA & $39(93 \%)$ & $11(52 \%)$ & 27 (82\%) & $51(68 \%)$ & 0.008 \\
\hline IB & $3(7 \%)$ & $6(29 \%)$ & $2(6 \%)$ & $12(16 \%)$ & \\
\hline II & $0(0 \%)$ & $4(19 \%)$ & $4(12 \%)$ & $12(16 \%)$ & \\
\hline \multicolumn{6}{|l|}{ LUS involvement, n (\%) } \\
\hline No & $34(81 \%)$ & $12(57 \%)$ & $21(64 \%)$ & $53(71 \%)$ & 0.19 \\
\hline Yes & $8(19 \%)$ & $9(43 \%)$ & $12(36 \%)$ & $22(29 \%)$ & \\
\hline $\begin{array}{l}\text { Percent myometrial involvement, mean } \\
\text { (SD) }\end{array}$ & $10(30)$ & $40(30)$ & $20(30)$ & $30(30)$ & 0.01 \\
\hline \multicolumn{6}{|l|}{ LVSI } \\
\hline Neg & 32 (76\%) & $18(86 \%)$ & 30 (91\%) & 57 (76\%) & 0.26 \\
\hline Pos & $10(24 \%)$ & $3(14 \%)$ & $3(9 \%)$ & $18(24 \%)$ & \\
\hline \multicolumn{6}{|l|}{ Dissection performed? n (\%) } \\
\hline No & $7(17 \%)$ & $2(10 \%)$ & $4(12 \%)$ & $8(11 \%)$ & 0.81 \\
\hline Yes & 35 (83\%) & 19 (90\%) & $29(88 \%)$ & $67(89 \%)$ & \\
\hline Number LN examined, median (range) & $12.5(0-72)$ & $7(0-64)$ & $10(0-45)$ & $21(0-56)$ & 0.003 \\
\hline $\begin{array}{l}\text { Number pelvic LN examined, median } \\
\text { (range) }\end{array}$ & $11.5(0-37)$ & $7(0-36)$ & $10(0-38)$ & $14(0-31)$ & 0.06 \\
\hline $\begin{array}{l}\text { Number para-aortic LN examined, median } \\
\text { (range) }\end{array}$ & $0(0-35)$ & $0(0-28)$ & $0(0-16)$ & $5(0-29)$ & $<0.001$ \\
\hline \multicolumn{6}{|l|}{$\begin{array}{l}\text { Para-aortic LN dissection performed? } \mathrm{n} \\
(\%)\end{array}$} \\
\hline No & $23(55 \%)$ & $15(71 \%)$ & $18(55 \%)$ & $19(25 \%)$ & $<0.001$ \\
\hline Yes & $19(45 \%)$ & $6(29 \%)$ & $15(45 \%)$ & 56 (75\%) & \\
\hline \multicolumn{6}{|l|}{ Type of surgery, n (\%) } \\
\hline Robotic & $21(50 \%)$ & $3(14 \%)$ & $9(27 \%)$ & 37 (49\%) & 0.005 \\
\hline Open & $21(50 \%)$ & $18(86 \%)$ & $24(73 \%)$ & 38 (51\%) & \\
\hline \multicolumn{6}{|l|}{ Omentectomy performed? n (\%) } \\
\hline No & $15(36 \%)$ & $14(67 \%)$ & $14(42 \%)$ & 24 (32\%) & 0.03 \\
\hline
\end{tabular}




\section{Cureus}

Yes

$27(64 \%)$

Type of RT

External

Brachy

Both

Peritoneal cytology, n (\%)
Negative

Positive

Not done

Recurrence, n (\%)

No

Yes

Vaginal recurrence, n (\%)

No

Yes

Pelvic recurrence, $\mathrm{n}(\%)$

No

Yes

Para-aortic recurrence, n (\%)

\section{No}

Yes

Distant recurrence, n (\%)

No

Yes

Vaginal only recurrence, $\mathrm{n}(\%)$

No

Yes

Pelvic only recurrence, $\mathrm{n}(\%)$

No

Yes

Vaginal/Pelvic recurrence, n (\%)
$5(24 \%)$

$3(14 \%)$

$13(62 \%)$

$35(83 \%)$

$4(10 \%)$

$3(7 \%)$

26 (62\%)

16 (38\%)

$34(81 \%)$

$8(19 \%)$

33 (79\%)

9 (21\%)

38 (90\%)

$4(10 \%)$

33 (79\%)

$9(21 \%)$

39 (93\%)

$3(7 \%)$

41 (91\%)

1 (9\%)

41 (98\%)

$1(2 \%)$

Para-aortic only recurrence, $\mathrm{n}(\%)$

No

Yes
$40(95 \%)$

$2(5 \%)$
$18(86 \%) \quad 24(73 \%)$

$60(80 \%)$

0.94

$2(10 \%) \quad 5(15 \%)$

$8(11 \%)$

$1(5 \%)$

$4(12 \%)$

$7(9 \%)$

$17(81 \%)$

$26(79 \%)$

$58(77 \%)$

0.21

$4(19 \%)$

7 (21\%)

$17(23 \%)$

$18(86 \%) \quad 29(88 \%)$

71 (95\%)

0.17

$3(14 \%)$

$4(12 \%)$

$4(5 \%)$

$21(100 \%)$

$28(85 \%)$

$61(81 \%)$

0.01

$0(0 \%)$

$5(15 \%)$

$14(19 \%)$

$21(100 \%)$

32 (97\%)

$66(88 \%)$

0.10

$0(0 \%)$

$1(3 \%)$

$9(12 \%)$

$19(90 \%) \quad 29(88 \%)$

$61(81 \%)$

0.25

$2(10 \%)$

$4(12 \%)$

14 (19\%)

$19(90 \%)$

$32(97 \%)$

$75(100 \%)$

0.17

$2(10 \%)$

$1(3 \%)$

$0(0 \%)$

$21(100 \%)$

$33(100 \%)$

73 (97\%)

0.14

$0(0 \%)$

$0(0 \%)$

2 (3\%)

$21(100 \%)$

31 (94\%)

75 (100\%)

0.14

$0(0 \%)$

$2(6 \%)$

$0(0 \%)$

$21(100 \%)$

$33(100 \%)$

74 (99\%)

1.00

$0(0 \%)$

$0(0 \%)$

$1(1 \%)$

\section{TABLE 1: Patient Characteristics Amongst Treatment Groups}

Abbreviations: BMI, Body mass index; CRT, Chemoradiotherapy; Chemo, Chemotherapy; LN, Lymph node; LUS, Lower uterine segment; FIGO, International Federation of Gynecology and Obstetrics; RT, Radiation therapy; SD, Standard deviation 


\section{Cureus}

The most common adjuvant chemotherapy regimen used in the study cohort was carboplatin (median dose area under curve six) and paclitaxel (median dose $175 \mathrm{mg} / \mathrm{m} 2$ ) given every three weeks for three to six cycles (median six cycles). Adjuvant RT was given with either pelvic external beam RT (EBRT) alone, vaginal cuff high-dose-rate brachytherapy (VB) alone, or a combination of pelvic EBRT and VB.

VB was delivered using an iridium-192 source. If receiving VB alone, patients were given 30 Gy in five fractions prescribed to the surface of the upper four $\mathrm{cm}$ of the vagina. If combined with EBRT, the prescription was $18 \mathrm{~Gy}$ in three fractions to the same target. VB treatments were given with one to two fractions per week. Pelvic EBRT was delivered with either intensity-modulated RT or 3-dimensional RT technique to a median dose of $45 \mathrm{~Gy}$ (range 44-50.4 Gy), using 1.8 to $2.0 \mathrm{~Gy}$ per fraction with daily treatments over five to six weeks. All patients completed the prescribed course of RT without significant treatment interruptions. No patients who underwent RT experienced grade three or higher toxicity from RT.

For overall recurrence, 44 women (26\%) developed cancer recurrence as detailed in Table 1. Five-year RFS for those with no adjuvant treatment was 55\% (95\% Confidence Interval (CI) 37\%-70\%), 84\% for RT alone (95\% CI 58\%-95\%), 68\% for chemo alone (95\% CI 44\%-84\%), and 73\% for CRT (95\% CI 60\%-83\%) ( $\mathrm{p}=0.13$ ) (Figure 1). Five-year DSS was $60 \%$ with observation ( $95 \%$ CI $42 \%-72 \%$ ), $94 \%$ with RT alone (95\% CI 66\%-99\%), $71 \%$ with chemo alone ( $95 \%$ CI $46 \%-86 \%$ ), and $81 \%$ with CRT (95\% CI 67\%-90\%) $(\mathrm{p}=0.03$ ) (Figure 2). Five-year OS was $56 \%$ with observation ( $95 \%$ CI $38 \%-70 \%$ ), $70 \%$ with RT alone (95\% CI $45 \%-85 \%$ ), $60 \%$ for chemo alone (95\% CI 38\%-76\%), and 76\% for CRT (95\% CI 61\%-85\%) ( $\mathrm{p}=0.114$ ) (Figure 3).

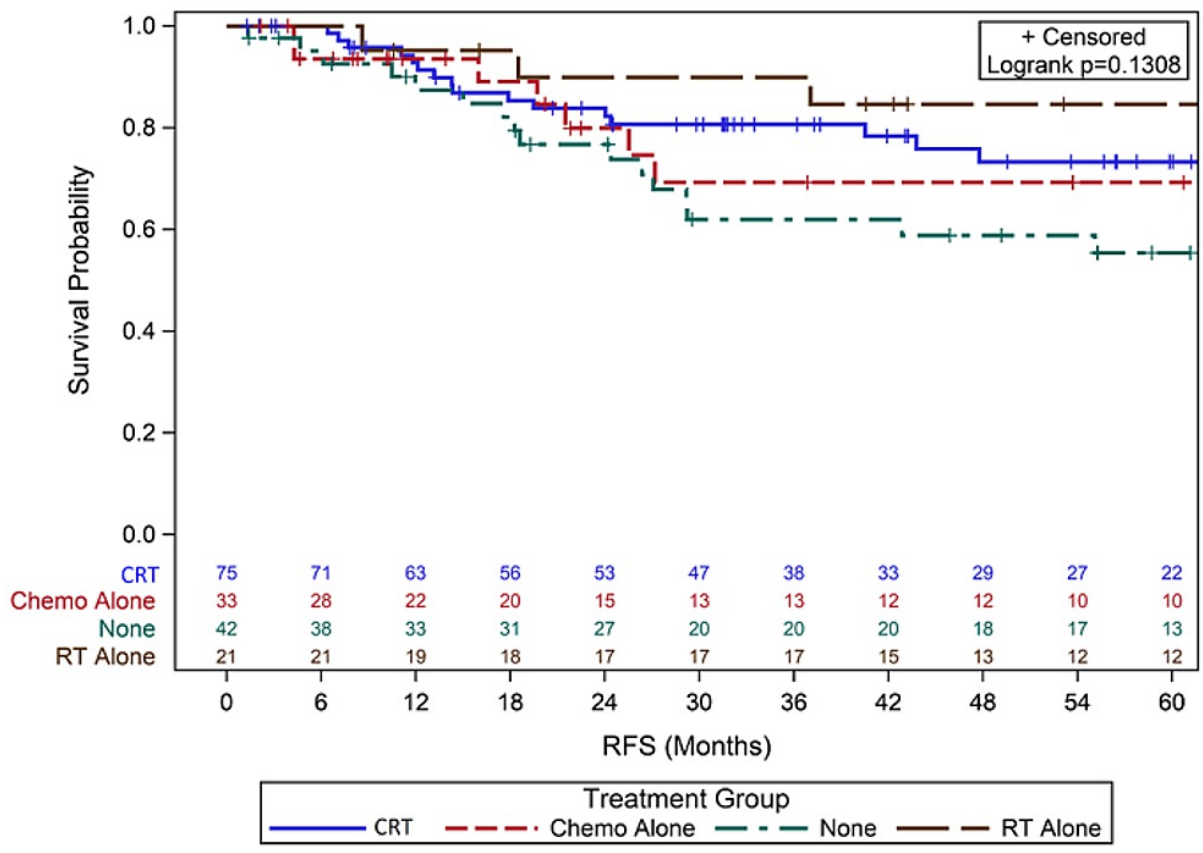

FIGURE 1: Kaplan-Meier Curves for Five-Year RFS

Abbreviations: Chemo, Chemotherapy; RFS, Recurrence-free survival; RT, Radiation therapy 


\section{Cureus}

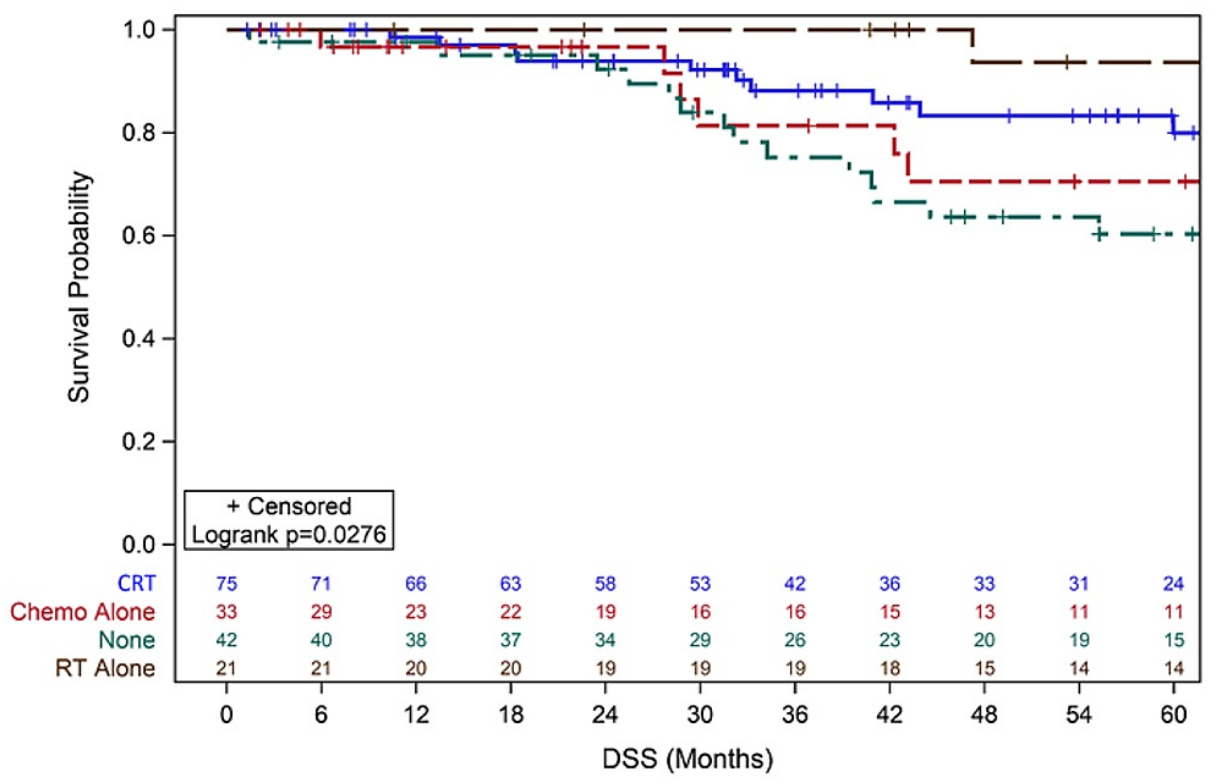

Treatment Group

CRT - - Chemo Alone - - - None - - RT Alone

FIGURE 2: Kaplan-Meier Curves for Five-Year DSS

Abbreviations: Chemo, Chemotherapy; DSS, Disease-specific survival; RT, Radiation therapy

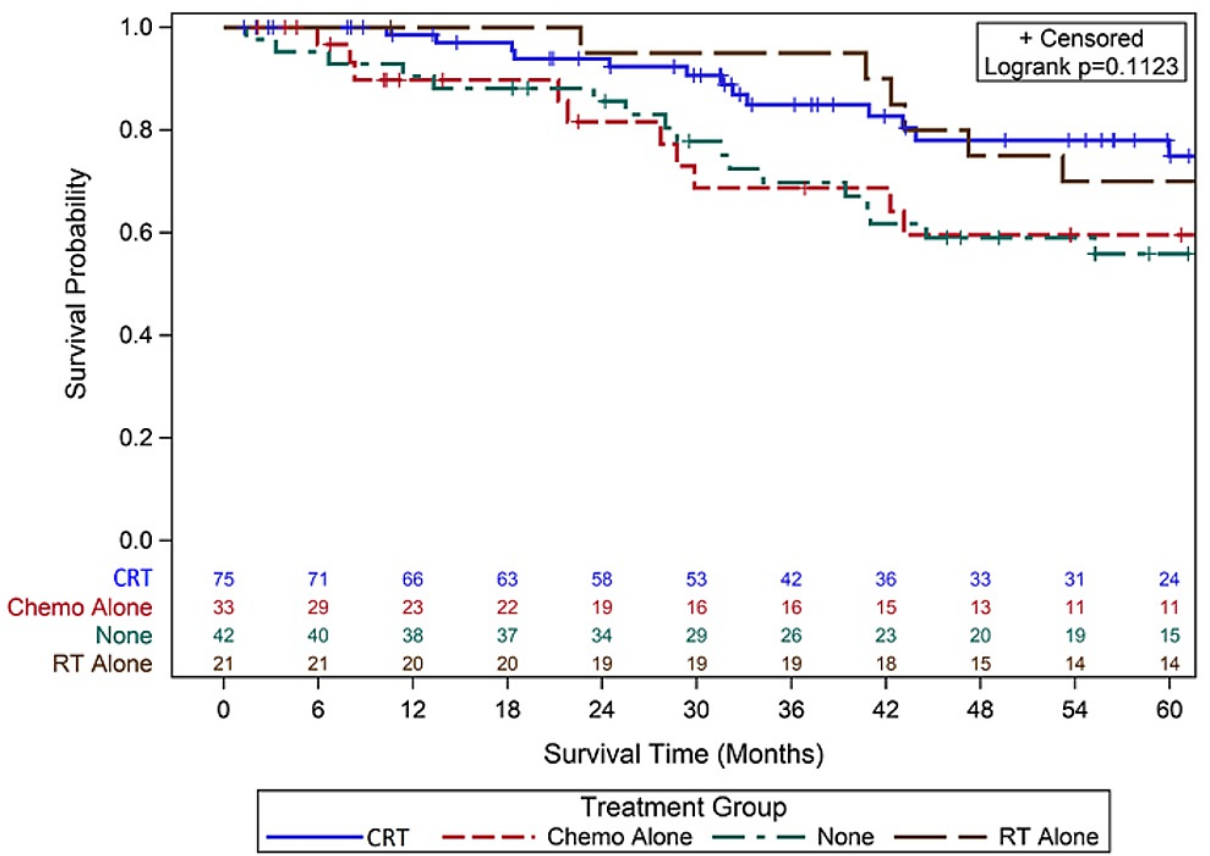

\section{FIGURE 3: Kaplan-Meier Curves for Five-Year OS}

Abbreviations: Chemo, Chemotherapy; OS, Overall survival; RT, Radiation therapy

Table 2 summarizes the variables included in the multivariate analysis (MVA) for RFS, DSS, and OS with their hazard ratios. The independent predictor of higher five-year RFS was negative peritoneal cytology. Independent predictors of higher five-year DSS included less myometrial invasion, negative peritoneal cytology, lack of lower uterine segment involvement, and the receipt of adjuvant CRT. Independent predictors of higher five-year OS were less myometrial invasion, lack of lower uterine segment involvement, and receipt of adjuvant CRT. 


\begin{tabular}{|c|c|c|c|c|c|c|c|c|c|}
\hline \multirow[b]{2}{*}{ Variables } & \multicolumn{3}{|c|}{ Overall Survival } & \multicolumn{3}{|c|}{ Recurrence-Free Survival } & \multicolumn{3}{|c|}{ Disease-Specific Survival } \\
\hline & $\mathrm{HR}$ & $95 \% \mathrm{Cl}$ of $\mathrm{HR}$ & p-value & $\mathrm{HR}$ & $95 \% \mathrm{Cl}$ of $\mathrm{HR}$ & p-value & $\mathrm{HR}$ & $95 \% \mathrm{Cl}$ of $\mathrm{HR}$ & p-value \\
\hline Charlson Score & 1.12 & $0.95-1.32$ & 0.17 & N/A & N/A & $\mathrm{N} / \mathrm{A}$ & $\mathrm{N} / \mathrm{A}$ & $\mathrm{N} / \mathrm{A}$ & $\mathrm{N} / \mathrm{A}$ \\
\hline Percent Myometrial Invasion & 10.09 & 2.84-35.88 & $<0.01$ & 2.46 & $0.77-7.83$ & 0.13 & 7.58 & $1.43-40.3$ & 0.017 \\
\hline \multicolumn{10}{|l|}{ Cytology } \\
\hline Negative vs Positive & 0.47 & $0.22-1.01$ & 0.052 & 0.44 & $0.20-0.96$ & 0.04 & 0.37 & $0.15-0.92$ & 0.03 \\
\hline LUS involvement & 1.98 & 1.03-3.82 & 0.041 & 0.71 & $0.37-1.35$ & 0.30 & 2.56 & $1.15-5.70$ & 0.021 \\
\hline Negative LVSI & 1.14 & $0.51-2.57$ & 0.75 & 0.48 & $0.23-1.01$ & 0.052 & 0.98 & $0.35-2.72$ & 0.97 \\
\hline \multicolumn{10}{|l|}{ Treatment Group } \\
\hline CRT vs RT & 1.38 & $0.50-3.81$ & 0.39 & N/A & N/A & N/A & 5.61 & $0.70-45.15$ & 0.11 \\
\hline Chemo vs RT & 3.00 & $1.01-8.94$ & 0.049 & N/A & N/A & N/A & 10.1 & $1.15-88.7$ & 0.04 \\
\hline Observation vs RT & 5.20 & $1.73-15.62$ & 0.003 & N/A & N/A & $\mathrm{N} / \mathrm{A}$ & 22.8 & $2.66-195.31$ & 0.004 \\
\hline
\end{tabular}

\section{TABLE 2: Multivariate Analysis for Survival Endpoints for the Study Cohort}

Abbreviations: HR: Hazard ratio; Cl, Confidence interval; LVSI, Lymphovascular space invasion; LUS, Lower uterine segment; CRT, Combined chemotherapy and radiation; RT, Radiation therapy; Chemo, chemotherapy; FIGO, International Federation of Gynecology and Obstetrics; N/A, Not applicable as it was not included in multivariate analysis

\section{Discussion}

As previously stated, the optimal adjuvant management for women with early USC remains controversial. Our study suggests that adjuvant RT with or without chemo provides statistically significant better uterine cancer-specific survival with a trend toward improving RFS and OS when compared to chemo alone and observation. This finding is in agreement with other studies that also showed improvement in survival outcomes with either RT alone or CRT [19-22,25-26]. Among the treatment groups in the DSS analysis, patients who received CRT and RT alone enjoyed the best five-year survival outcomes (81\% and 94\%, respectively). Additionally, our survival outcomes in patients who received RT as part of their treatment course are comparable to other prospective and retrospective studies [16,25-26].

As shown in our analysis, chemo alone was not found to be a significant predictor of improved OS, RFS, or DSS. This result has also been found in other studies, including a subset analysis of a randomized trial comparing adjuvant RT with or without sequential chemo. The results showed that chemo did not have an effect on progression-free survival or OS in patients with any stage serous or clear cell carcinoma [27]. Additionally, a study by Barney et al. looked at outcomes of early-stage serous and clear cell patients who received VB and showed that chemo did not improve survival outcomes [28].

Our study findings are also in agreement with the Gynecologic Oncology Group-249 (GOG-249) study, a large randomized trial that compared pelvic RT alone versus VB plus three cycles of chemo in women with stage III endometrial carcinoma with endometrioid and non-endometrioid histologies. It showed no significant difference in terms of survival outcomes in those who received three cycles of chemo with VB compared to those who received pelvic RT alone, although the proportion of women with USC in this study was small [29].

Our results contrast with those seen in the Post-Operative Radiation Therapy in Endometrial Cancer-3 (PORTEC-3) study, which showed that CRT improved survival outcomes compared to RT alone for earlystage USC, suggesting that chemo plays an important role in USC. However, PORTEC-3 included women with advanced-stage USC and excluded women with non-myoinvasive FIGO stage IA disease [24]. Twenty percent of our study cohort had a non-myoinvasive disease, and $75 \%$ of our patients were FIGO stage IA. It is also possible that our study is underpowered to detect a positive impact of adjuvant chemo on survival endpoints.

A limitation of this study is the selection bias inherent to a retrospective analysis, which may explain why some of our findings differ from prospective studies. Although the sample size of this study is relatively small compared to some of the larger prospective trials that have been mentioned, this analysis is one of the largest single-institution studies to date that examines outcomes solely in stage I-II USC. This analysis was able to confirm serous histology by an expert gynecologic pathologist in a respectable sample size. 
Furthermore, the study groups were overall well-balanced, which strengthens the findings of this study.

\section{Conclusions}

The optimal adjuvant management strategy for early-stage USC remains controversial. This singleinstitution retrospective study evaluating the impact of different adjuvant management strategies on earlystage USC suggests that adjuvant RT with or without chemo improved DSS with a trend towards improved OS and RFS. Predictors of improved DSS were less myometrial invasion, negative peritoneal cytology, lack of lower uterine segment involvement, and receipt of adjuvant CRT. Further prospective trials will be needed to determine the optimal combination of chemo and RT for patients with this relatively rare histology.

\section{Additional Information}

\section{Disclosures}

Human subjects: Consent was obtained or waived by all participants in this study. Henry Ford Institutional Review Board issued approval 4645. The Henry Ford IRB approved the protocol that was associated with conducting this retrospective study. Given the retrospective nature of this project, informed consent was waived. Animal subjects: All authors have confirmed that this study did not involve animal subjects or tissue. Conflicts of interest: In compliance with the ICMJE uniform disclosure form, all authors declare the following: Payment/services info: All authors have declared that no financial support was received from any organization for the submitted work. Financial relationships: All authors have declared that they have no financial relationships at present or within the previous three years with any organizations that might have an interest in the submitted work. Other relationships: All authors have declared that there are no other relationships or activities that could appear to have influenced the submitted work.

\section{References}

1. Siegel RL, Miller KD, Jemal A: Cancer statistics, 2019. CA: Cancer J Clin. 2019, 69:7-34. 10.3322/caac.21551

2. Creutzberg CL, Nout RA, Lybeert ML, et al.: Fifteen-year radiotherapy outcomes of the randomized PORTEC-1 trial for endometrial carcinoma. Int J Radiat Oncol Biol Phys. 2011, 81:631-638. 10.1016/j.ijrobp.2011.04.013

3. Fader AN, Santin AD, Gehrig PA: Early stage uterine serous carcinoma: management updates and genomic advances. Gynecol Oncol. 2013, 129:244-250. 10.1016/j.ygyno.2013.01.004

4. Fader AN, Java J, Tenney M, et al.: Impact of histology and surgical approach on survival among women with early-stage, high-grade uterine cancer. Gynecol Oncol. 2016, 143:460-465. 10.1016/j.ygyno.2016.10.016

5. Cirisano FD, Robboy SJ, Dodge RK, et al.: The outcome of stage I-II clinically and surgically staged papillary serous and clear cell endometrial cancers when compared with endometrioid carcinoma. Gynecol Oncol. 2000, 77:55-65. 10.1006/gyno.2000.5737

6. Matthews RP, Hutchinson-Colas J, Maiman M, et al.: Papillary serous and clear cell type lead to poor prognosis of endometrial carcinoma in black women. Gynecol Oncol. 1997, 65:206-212. 10.1006/gyno.1997.4617

7. Fader AN, Drake RD, O'Malley DM, et al.: Platinum/taxane-based chemotherapy with or without radiation therapy favorably impacts survival outcomes in stage I uterine papillary serous carcinoma. Cancer. 2009, 115:2119-2127. 10.1002/cncr.24247

8. Thomas MB, Mariani A, Cliby WA, Keeney GA, Podratz KC, Dowdy SC: Role of systematic lymphadenectomy and adjuvant therapy in stage I uterine papillary serous carcinoma. Gynecol Oncol. 2007, 107:186-189. 10.1016/j.ygyno.2007.05.044

9. Havrilesky LJ, Secord AA, Bae-Jump V, et al.: Outcomes in surgical stage I uterine papillary serous carcinoma. Gynecol Oncol. 2007, 105:677-682. 10.1016/j.ygyno.2007.01.041

10. Boruta DM, Gehrig PA, Groben PA, Bae-Jump V, Boggess JF, Fowler WC Jr, Van Le L: Uterine serous and grade 3 endometrioid carcinomas. Is there a survival difference?. Cancer. 2004, 101:2214-2221. $10.1002 /$ cncr.20645

11. Ueda SM, Kapp DS, Cheung MK, et al.: Trends in demographic and clinical characteristics in women diagnosed with corpus cancer and their potential impact on the increasing number of deaths. Am J Obstet Gynecol. 2008, 218:211-216. 10.1016/j.ajog.2007.08.075

12. Zhong X, Wang J, Kaku T, Wang Z, Li X, Wei L: Prognostic factors of uterine serous carcinoma-a multicenter study. Int J Gynecol Cancer. 2018, 28:1138-1144. 10.1097/igc.0000000000001272

13. Park JY, Nam JH, Kim YT, et al.: Poor prognosis of uterine serous carcinoma compared with grade 3 endometrioid carcinoma in early stage patients. Virchows Arch. 2013, 462:289-296. 10.1007/s00428-0131382-8

14. Velker V, D'Souza D, Prefontaine M, McGee J, Leung E: Role of adjuvant therapy for stage IA serous and clear cell uterine cancer: is observation a valid strategy?. Int J Gynecol Cancer. 2016, 26:491-496. 10.1097/igc.0000000000000643

15. Tate K, Yoshida H, Ishikawa M, Uehara T, Ikeda SI, Hiraoka N, Kato T: Prognostic factors for patients with early-stage uterine serous carcinoma without adjuvant therapy. J Gynecol Oncol. 2018, 29:e34. 10.3802/igo.2018.29.e34

16. Frimer M, Miller EM, Shankar V, et al.: Adjuvant pelvic radiation "sandwiched" between paclitaxel/carboplatin chemotherapy in women with completely resected uterine serous carcinoma: longterm follow-up of a prospective phase 2 trial. Int J Gynecol Cancer. 2018, 28:1781-1788. 10.1097/igc.0000000000001359

17. Mukerji B, Baptiste C, Chen L, et al.: Racial disparities in young women with endometrial cancer . Gynecol Oncol. 2018, 148:527-534. 10.1016/j.ygyno.2017.12.032 
18. Nasioudis D, Roy AG, Ko EM, et al.: Adjuvant treatment for patients with FIGO stage I uterine serous carcinoma confined to the endometrium. Int J Gynecol Cancer. 2020, 30:1089-1094. 10.1136/ijgc-2020001379

19. Cham S, Huang Y, Tergas AI, et al.: Utility of radiation therapy for early-stage uterine papillary serous carcinoma. Gynecol Oncol. 2017, 145:269-276. 10.1016/j.ygyno.2017.03.003

20. Hong JC, Foote J, Broadwater G, Gaillard S, Havrilesky LJ, Chino JP: Impact of chemotherapy and radiotherapy on management of early stage clear cell and papillary serous carcinoma of the uterus. Int J Gynecol Cancer. 2017, 27:720-729. 10.1097/igc.0000000000000926

21. Qu XM, Velker VM, Leung E, et al.: The role of adjuvant therapy in stage IA serous and clear cell uterine cancer: a multi-institutional pooled analysis. Gynecol Oncol. 2018, 149:283-290. 10.1016/j.ygyno.2018.03.002

22. Lin Y, Zhou J, Cheng Y, Zhao L, Yang Y, Wang J: Comparison of survival benefits of combined chemotherapy and radiotherapy versus chemotherapy alone for uterine serous carcinoma: a meta-analysis. Int J Gynecol Cancer. 2017, 27:93-101. 10.1097/igc.0000000000000856

23. Yechieli R, Rasool N, Robbins JR, Cogan CM, Elshaikh MA: Adjuvant radiation therapy for patients with type II endometrial carcinoma: impact on tumor recurrence and survival. Int J Gynecol Cancer. 2013, 23:763-768. 10.1097/IGC.0b013e31828b15cb

24. de Boer SM, Powell ME, Mileshkin L, et al.: Adjuvant chemoradiotherapy versus radiotherapy alone in women with high-risk endometrial cancer (PORTEC-3): patterns of recurrence and post-hoc survival analysis of a randomised phase 3 trial. Lancet Oncol. 2019, 20:1273-1285. 10.1016/s1470-2045(19)30395-X

25. Modh A, Burmeister C, Munkarah AR, Elshaikh MA: External pelvic and vaginal irradiation vs. vaginal irradiation alone as postoperative therapy in women with early stage uterine serous carcinoma: results of a National Cancer Database analysis. Brachytherapy. 2017, 16:841-846. 10.1016/j.brachy.2017.04.237

26. Desai NB, Kiess AP, Kollmeier MA, Abu-Rustum NR, Makker V, Barakat RR, Alektiar KM: Patterns of relapse in stage I-II uterine papillary serous carcinoma treated with adjuvant intravaginal radiation (IVRT) with or without chemotherapy. Gynecol Oncol. 2013, 131:604-608. 10.1016/j.ygyno.2013.09.019

27. Hogberg T, Signorelli M, de Oliveira CF, et al.: Sequential adjuvant chemotherapy and radiotherapy in endometrial cancer--results from two randomised studies. Eur J Cancer. 2010, 46:2422-2431. 10.1016/j.ejca.2010.06.002

28. Barney BM, Petersen IA, Mariani A, Dowdy SC, Bakkum-Gamez JN, Haddock MG: The role of vaginal brachytherapy in the treatment of surgical stage I papillary serous or clear cell endometrial cancer. Int J Radiat Oncol Biol Phys. 2013, 85:109-115. 10.1016/j.ijrobp.2012.03.011

29. Randall ME, Filiaci V, McMeekin DS, et al.: Phase III trial: adjuvant pelvic radiation therapy versus vaginal brachytherapy plus paclitaxel/carboplatin in high-intermediate and high-risk early stage endometrial cancer. J Clin Oncol. 2019, 37:1810-1818. 10.1200/jco.18.01575 